PROCEEDINGS OF THE

AMERICAN MATHEMATICAL SOCIETY

Volume 139, Number 1, January 2011, Pages 99-110

S 0002-9939(2010)10478-1

Article electronically published on July 13, 2010

\title{
TWISTING THE QUANTUM GRASSMANNIAN
}

\author{
S. LAUNOIS AND T. H. LENAGAN
}

(Communicated by Martin Lorenz)

\begin{abstract}
In contrast to the classical and semiclassical settings, the Coxeter element $(12 \ldots n)$ which cycles the columns of an $m \times n$ matrix does not determine an automorphism of the quantum grassmannian. Here, we show that this cycling can be obtained by means of a cocycle twist. A consequence is that the torus invariant prime ideals of the quantum grassmannian are permuted by the action of the Coxeter element $(12 \ldots n)$. We view this as a quantum analogue of the recent result of Knutson, Lam and Speyer, where the Lusztig strata of the classical grassmannian are permuted by $(12 \ldots n)$.
\end{abstract}

\section{INTRODUCTION}

The symmetric group $S_{n}$ acts on the complex grassmannian $G(m, n)$ by permuting the columns of an $m \times n$ matrix that determine a point in $G(m, n)$. If one restricts oneself to considering the totally nonnegative grassmannian $G(m, n)^{\mathrm{tnn}}$, this is no longer true. However, Postnikov, [15, Remark 3.3], notes that the cycle $c=(12 \ldots n)$ acts on the totally nonnegative grassmannian. Recently, Knutson, Lam and Speyer, [10, showed that the Lusztig strata of the classical grassmannian are permuted by $(12 \ldots n)$. In fact this invariance property is even stronger. Indeed, Goodearl and Yakimov, 6, have found a Poisson interpretation of the Lusztig strata: they coincide with the $\mathcal{H}$-orbits of symplectic leaves of $G(m, n)$, where $\mathcal{H}$ is an $n$-dimensional algebraic torus. Recently Yakimov, 16], showed that the Coxeter element $c$ induces a Poisson automorphism of $G(m, n)$. As a consequence he showed that the $\mathcal{H}$-orbits of symplectic leaves of $G(m, n)$ are permuted by $c$; this gives a Poisson geometric proof of the Knutson, Lam and Speyer result.

In view of the close connections that have been discovered between totally nonnegative matrices, the standard Poisson matrix variety and quantum matrices (see, for example, [3, 4]) and between the totally nonnegative grassmannian and the quantum grassmannian (see, for example, 11]), one might expect that the cycle $c$ produces an automorphism of the quantum grassmannian. This is not the case; see Example 3.1 below. With this in mind, one wonders what the analogous result should be. Here, we provide an answer: there is a 2-cocyle which can be used to twist the quantum grassmannian; the resulting twisted algebra is again isomorphic

Received by the editors October 1, 2009 and, in revised form, March 15, 2010.

2010 Mathematics Subject Classification. Primary 16T20, 16P40, 16S38, 17B37, 20G42.

Key words and phrases. Quantum matrices, quantum grassmannian, cocycle twist, noncommutative dehomogenisation.

The research of the first author was supported by a Marie Curie European Reintegration Grant within the $7^{\text {th }}$ European Community Framework Programme.

(C)2010 American Mathematical Society Reverts to public domain 28 years from publication 
to the quantum grassmannian, and the effect of the twist on a generating quantum minor $I$ is to produce (a scalar multiple of) the quantum minor obtained by letting the cycle $c$ act on the indices of $I$. A consequence of this result is that the torus invariant prime ideals of the quantum grassmannian are permuted by the cycle $(12 \ldots n)$; see Corollary 6.2. We view this as a quantum analogue of the Knutson, Lam and Speyer result.

\section{BASIC DEFINITIONS}

In this section, we will give the basic definitions of the objects that interest us in this paper and recall several results that we need in our proofs. Throughout, $\mathbb{K}$ will denote a base field, we set $\mathbb{K}^{*}:=\mathbb{K} \backslash\{0\}, q$ will be a nonzero element of $\mathbb{K}$, and $m$ and $n$ will denote positive integers with $m<n$. Moreover, we assume that there exists $p \in \mathbb{K}$ such that $p^{m}=q^{2}$.

The quantisation of the coordinate ring of the affine variety $M_{m, n}$ of $m \times n$ matrices with entries in $\mathbb{K}$ is denoted by $\mathcal{O}_{q}\left(M_{m, n}\right)$. It is the $\mathbb{K}$-algebra generated by $m n$ indeterminates $X_{i j}$, with $1 \leq i \leq m$ and $1 \leq j \leq n$, subject to the relations:

$$
\begin{array}{ll}
X_{i j} X_{i l}=q X_{i l} X_{i j}, & \text { for } 1 \leq i \leq m, \text { and } 1 \leq j<l \leq n ; \\
X_{i j} X_{k j}=q X_{k j} X_{i j}, & \text { for } 1 \leq i<k \leq m, \text { and } 1 \leq j \leq n ; \\
X_{i j} X_{k l}=X_{k l} X_{i j}, & \text { for } 1 \leq k<i \leq m, \text { and } 1 \leq j<l \leq n ; \\
X_{i j} X_{k l}-X_{k l} X_{i j}=\left(q-q^{-1}\right) X_{i l} X_{k j}, & \text { for } 1 \leq i<k \leq m, \text { and } 1 \leq j<l \leq n .
\end{array}
$$

An index pair is a pair $(I, J)$ such that $I \subseteq\{1, \ldots, m\}$ and $J \subseteq\{1, \ldots, n\}$ are subsets with the same cardinality. Hence, an index pair is given by an integer $t$ such that $1 \leq t \leq m$ and ordered sets $I=\left\{i_{1}<\cdots<i_{t}\right\} \subseteq\{1, \ldots, m\}$ and $J=\left\{j_{1}<\cdots<j_{t}\right\} \subseteq\{1, \ldots, n\}$. To any such index pair we associate the quantum minor

$$
[I \mid J]=\sum_{\sigma \in S_{t}}(-q)^{\ell(\sigma)} X_{i_{\sigma(1)} j_{1}} \cdots X_{i_{\sigma(t)} j_{t}} .
$$

Definition 2.1. The quantisation of the homogeneous coordinate ring of the grassmannian of $m$-dimensional subspaces of $\mathbb{K}^{n}$, denoted by $\mathcal{O}_{q}(G(m, n))$ and informally referred to as the $(m \times n)$ quantum grassmannian, is the subalgebra of $\mathcal{O}_{q}\left(M_{m, n}\right)$ generated by the $m \times m$ quantum minors.

A maximal (that is, $m \times m$ ) quantum minor in $\mathcal{O}_{q}\left(M_{m, n}\right)$ corresponds to an index pair $[\{1, \ldots, m\} \mid J]$ with $J=\left\{j_{1}, \ldots, j_{m}\right\} \subseteq\{1, \ldots, n\}$. We call such $J$ index sets and denote the corresponding quantum minor by $[J]$ or $\left[j_{1}, \ldots, j_{m}\right]$ in what follows. Thus, such a $[J]$ is a generator of $\mathcal{O}_{q}(G(m, n))$.

When writing an $m \times m$ quantum minor in $\mathcal{O}_{q}(G(m, n))$, we will use the convention that if a column index $j$ is greater than $n$, then $j$ is to be read as $j-n$. For example, in $\mathcal{O}_{q}(G(2,4))$ the quantum minor specified by [45] is the quantum minor [14]. In order to stress this point, we will use the convention that $\widetilde{j}$ is the integer in the set $\{1, \ldots, n\}$ that is congruent to $j$ modulo $n$.

A quantum minor $\widetilde{[i}, \widetilde{i+1}, \ldots, i \widetilde{+m-1}]$ is said to be a consecutive quantum minor of $\mathcal{O}_{q}(G(m, n))$. Recalling the convention above, we see that there are four consecutive quantum minors in $\mathcal{O}_{q}(G(2,4))$ : they are [12], [23], [34] and [ $\left.\widetilde{4} \widetilde{5}\right]=[14]$. More generally, $\mathcal{O}_{q}(G(m, n))$ has $n$ consecutive minors.

Two maximal quantum minors $[I]$ and $[J]$ are said to quasi-commute if there is an integer $c$ such that $[I][J]=q^{c}[J][I]$. Recall that an element $u$ of a ring $R$ is said 
to be a normal element if $u R=R u$, in which case $u R$ is a two-sided ideal. The following lemma, first obtained in [9, Lemma 3.7], shows that consecutive quantum minors quasi-commute with all maximal quantum minors.

Lemma 2.2. Let $\widetilde{i}, \widetilde{i+1}, \ldots, i \widetilde{+m-1}]$ be a consecutive quantum minor in the quantum grassmannian $\mathcal{O}_{q}(G(m, n))$. Then $\left.\widetilde{i}, \widetilde{i+1}, \ldots, i \widetilde{+m-1}\right]$ quasi-commutes with each of the generating quantum minors of $\mathcal{O}_{q}(G(m, n))$. In particular, each consecutive quantum minor is a normal element of $\mathcal{O}_{q}(G(m, n))$.

A consequence of this result is that the powers of a consecutive quantum minor form an Ore set in the noetherian domain $\mathcal{O}_{q}(G(m, n))$, and so it is possible to invert a consecutive quantum minor in a localisation.

In order to facilitate computations, we need a version of the Quantum Muir's Law of Extensible Minors. This result was first obtained by Krob and Leclerc, 9, Theorem 3.4], with a proof involving quasi-determinants. The version below, which is sufficient for our needs, is taken from [13, Proposition 1.3] and is adapted for use in the quantum grassmannian.

Proposition 2.3. Let $I_{s}, J_{s}$, for $1 \leq s \leq d$, be m-element subsets of $\{1, \ldots, n\}$ and let $c_{s} \in \mathbb{K}$ be such that $\sum_{s=1}^{d} c_{s}\left[I_{s}\right]\left[J_{s}\right]=0$ in $\mathcal{O}_{q}(G(m, n))$. Suppose that $P$ is a subset of $\{1, \ldots, n\}$ such that $\left(\bigcup_{s=1}^{d} I_{s}\right) \cup\left(\bigcup_{s=1}^{d} J_{s}\right) \subseteq P$ and let $\bar{P}$ denote $\{1, \ldots, n\} \backslash P$. Then

$$
\sum_{s=1}^{d} c_{s}\left[I_{s} \sqcup \bar{P}\right]\left[J_{s} \sqcup \bar{P}\right]=0
$$

holds in $\mathcal{O}_{q}\left(G\left(m^{\prime}, n\right)\right)$, where $m^{\prime}=m+\# \bar{P}$.

This result is used, for example, when it is necessary to write a commutation relation between two maximal quantum minors $[I]$ and $[J]$, say. The usefulness of the result is that one may delete the common members of the index pairs $I$ and $J$ to establish the commutation relation.

\section{CyCling DOES NOT INDUCE AN AUTOMORPhism}

In contrast to the classical and semiclassical settings, the cycle $(12 \ldots n)$ does not act as an automorphism on the quantum grassmannian. We show this here by considering $\mathcal{O}_{q}(G(2,4))$.

First, we summarize the commutation relations and the quantum Plücker relations for $\mathcal{O}_{q}(G(2,4))$, which can easily be obtained from the defining relations of quantum matrices:

$$
[i j][i k]=q[i k][i j], \quad[i k][j k]=q[j k][i k], \quad \text { for } i<j<k
$$

and

$$
[14][23]=[23][14], \quad[12][34]=q^{2}[34][12], \quad[13][24]=[24][13]+\left(q-q^{-1}\right)[14][23] .
$$

There is also a quantum Plücker relation [12] [34] $-q[13][24]+q^{2}[14][23]=0$. This relation may be rewritten as [34] [12] $-q^{-1}[24][13]+q^{-2}[23][14]=0$, and one can also check that [13] [24] $=q^{2}[24][13]+\left(q^{-1}-q\right)[12][34]$. 
Example 3.1. Let $\theta[i j]:=[i+1, j+1]$, with the convention that $\theta(4)=1$; that is, we work modulo 4 , and $\theta$ is cycling the indices of quantum minors:

$$
\theta[i j]=\widetilde{[c(i)}, \widetilde{c(j)}],
$$

where $c$ denotes the cycle (1234).

In the classical case, $\theta$ induces an isomorphism, and this is also the case in the Poisson setting, 16.

However, $\theta$ does not induce an automorphism of $\mathcal{O}_{q}(G(2,4))$, since, for example, the first quantum Plücker relation above is not preserved: if we assume that $\theta$ induces an automorphism, then we calculate

$$
0=\theta(0)=\theta\left([12][34]-q[13][24]+q^{2}[14][23]\right)=[23][14]-q[24][13]+q^{2}[12][34] .
$$

However, one can check that $[23][14]-q[24][13]+q^{2}[12][34] \neq 0$. For, suppose that $[23][14]-q[24][13]+q^{2}[12][34]=0$; then $[23][14]-q[24][13]+q^{4}[34][12]=0$. However, from the second version above of the quantum Plücker relation, we know that $[14][23]-q[24][13]+q^{2}[34][12]=0$. Subtract one of these equations from the other and note that [14][23] $=[23][14]$ to obtain $\left(q^{4}-q^{2}\right)[34][12]=0$, a contradiction, provided that $q^{2} \neq 1$.

\section{Dehomogenisation at a COnSECutive minor}

Explicit calculations in the quantum grassmannian can be difficult due to the awkward defining relations (quantum Plücker relations). For this reason, it is often useful to transfer to an overring where the defining relations are simpler. This can be achieved by localising at any consecutive quantum minor, and this leads to consideration of the noncommutative dehomogenisation isomorphism for an arbitrary consecutive quantum minor.

Set $M_{\alpha}:=\left\{\widetilde{\alpha}, \widetilde{\alpha+1}, \ldots, \alpha \widetilde{+m-1}\right.$. Now, $\left[M_{\alpha}\right]$ is a normal element in $\mathcal{O}_{q}(G(m, n))$, by Lemma 2.2 , so we may form the localisation $\mathcal{O}_{q}(G(m, n))\left[\left[M_{\alpha}\right]^{-1}\right]$. In $\mathcal{O}_{q}(G(m, n))\left[\left[M_{\alpha}\right]^{-1}\right]$ set

$$
x_{i j}:=\left[M_{\alpha} \cup\{j+\widetilde{\alpha+m}-1\} \backslash\{\alpha \widetilde{+m}-i\}\right]\left[M_{\alpha}\right]^{-1}
$$

for $1 \leq i \leq m$ and $1 \leq j \leq n-m$.

Theorem 4.1. The subalgebra $\mathbb{K}\left[x_{i j}\right]$ of $\mathcal{O}_{q}(G(m, n))\left[\left[M_{\alpha}\right]^{-1}\right]$ is a q-quantum matrix algebra; that is, $\mathbb{K}\left[x_{i j}\right]$ is isomorphic to $\mathcal{O}_{q}\left(M_{m, n-m}\right)$ by an isomorphism that sends $x_{i j}$ to $X_{i j}$. Moreover there is an isomorphism

$$
\phi_{\alpha}: \mathcal{O}_{q}(G(m, n))\left[\left[M_{\alpha}\right]^{-1}\right] \longrightarrow \mathbb{K}\left[x_{i j}\right]\left[y_{\alpha}^{ \pm 1} ; \sigma_{\alpha}\right]
$$

where $\sigma_{\alpha}$ is the automorphism of the quantum matrix algebra $\mathbb{K}\left[x_{i j}\right]$ defined by $\sigma_{\alpha}\left(x_{i j}\right)=\left[M_{\alpha}\right] x_{i j}\left[M_{\alpha}\right]^{-1}$. Under this isomorphism, $y_{\alpha}=\phi_{\alpha}\left(\left[M_{\alpha}\right]\right)$.

Proof. The fact that $\mathbb{K}\left[x_{i j}\right]$ is a quantum matrix algebra is established in [14, Theorem 3.2]. The inclusion $\rho_{\alpha}: \mathbb{K}\left[x_{i j}\right] \longrightarrow \mathcal{O}_{q}(G(m, n))\left[\left[M_{\alpha}\right]^{-1}\right]$ extends to a homomorphism $\rho_{\alpha}: \mathbb{K}\left[x_{i j}\right]\left[y_{\alpha}^{ \pm 1} ; \sigma_{\alpha}\right] \longrightarrow \mathcal{O}_{q}(G(m, n))\left[\left[M_{\alpha}\right]^{-1}\right]$, with $\rho_{\alpha}\left(y_{\alpha}\right)=\left[M_{\alpha}\right]$, by the universal property of skew polynomial extensions. The fact that the extension $\rho_{\alpha}$ is an isomorphism follows from [14, Lemma 3.1] and the dehomogenisation isomorphism [7, Lemma 3.1]. Now, set $\phi_{\alpha}=\rho_{\alpha}^{-1}$. 
Next, we need to calculate the effect of $\phi_{\alpha}$ on generating quantum minors of $\mathcal{O}_{q}(G(m, n))$.

Let $I$ be an $m$-element subset of $\{1, \ldots, n\}$. For a fixed $\alpha$, set $I_{r}:=I \cap M_{\alpha}$ and $I_{c}:=I \backslash I_{r}$ so that $I=I_{r} \sqcup I_{c}$ (the notation is chosen because $I_{r}$ will give information about the row set of the image of $[I]$ and $I_{c}$ will give information about the column set).

To simplify the notation somewhat, if $N$ is a subset of integers and $i$ is an integer, then $i+N=\{i+k \mid k \in N\}$.

Corollary 4.2. Let $[I]$ be a generating quantum minor of $\mathcal{O}_{q}(G(m, n))$. Then

$$
\phi_{\alpha}([I])=\left[(\alpha+m)-\left(M_{\alpha} \backslash I_{r}\right) \mid I_{c}-(\alpha+m-1)\right] y_{\alpha} .
$$

Proof. By using [14, Proposition 4.3], we see that for a quantum minor $[I \mid J]$ of the quantum matrix algebra $\mathbb{K}\left[x_{i j}\right]$,

$$
\rho_{\alpha}([I \mid J])=\left[M_{\alpha} \backslash((\alpha+m)-I) \sqcup((\alpha+m-1)+J)\right]\left[M_{\alpha}\right]^{-1} .
$$

As $\phi_{\alpha}=\rho_{\alpha}^{-1}$, the claim will be established once we show that

$$
\rho_{\alpha}\left(\left[(\alpha+m)-\left(M_{\alpha} \backslash I_{r}\right) \mid I_{c}-(\alpha+m-1)\right] \cdot y_{\alpha}\right)=[I] .
$$

Now,

$$
\begin{aligned}
& \rho_{\alpha}\left(\left[(\alpha+m)-\left(M_{\alpha} \backslash I_{r}\right) \mid I_{c}-(\alpha+m-1)\right] \cdot y_{\alpha}\right) \\
&= {\left[M_{\alpha} \backslash((\alpha+m)\right.} \\
&\left.\quad-\left((\alpha+m)-\left(M_{\alpha} \backslash I_{r}\right)\right) \sqcup\left((\alpha+m-1)+\left(I_{c}-(\alpha+m-1)\right)\right)\right] \\
& \quad= {\left[M_{\alpha} \backslash\left(M_{\alpha} \backslash I_{r}\right) \sqcup I_{c}\right] } \\
& \quad= {\left[I_{r} \sqcup I_{c}\right]=[I], }
\end{aligned}
$$

as required.

We shall need to use the isomorphisms $\phi_{\alpha}$ and $\rho_{\alpha}$ of Theorem 4.1 in the two cases $\alpha=1$ and $\alpha=2$. The next two results record the action of $\sigma_{1}$ and $\sigma_{2}$.

Lemma 4.3. For $1 \leq i \leq m$ and $1 \leq j \leq n-m$,

$$
\sigma_{1}\left(x_{i j}\right)=q x_{i j} .
$$

Consequently, $y_{1} x_{i j}=q x_{i j} y_{1}$ for $1 \leq i \leq m$ and $1 \leq j \leq n-m$.

Proof. In order to calculate the commutation relation between $x_{i j}$ and $y_{1}$, we need to consider the commutation relation between $x_{i j}$ and $\left[M_{1}\right]$. This will be the same as the commutation relation between $x_{i j}\left[M_{1}\right]$ and $\left[M_{1}\right]$. Set $N:=\{1, \ldots, m\} \backslash\{m+$ $1-i\}$. Then $x_{i j}\left[M_{1}\right]=[N \cup\{j+m\}]$ and $\left[M_{1}\right]=[N \cup\{m+1-i\}]$. Note that $m+1-i<j+m$, so that $[m+1-i][j+m]=q[j+m][m+1-i]$ in $\mathcal{O}_{q}(G(1, n))$. By using Proposition [2.3, it follows that $\left[M_{1}\right]\left(x_{i j}\left[M_{1}\right]\right)=q\left(x_{i j}\left[M_{1}\right]\right)\left[M_{1}\right]$. Hence, $\left[M_{1}\right] x_{i j}=q x_{i j}\left[M_{1}\right]$, and so $\sigma_{1}\left(x_{i j}\right)=q x_{i j}$ and $y_{1} x_{i j}=q x_{i j} y_{1}$, as claimed.

Lemma 4.4. For $1 \leq i \leq m$ and $1 \leq j<n-m$,

$$
\sigma_{2}\left(x_{i j}\right)=q x_{i j},
$$

while $\sigma_{2}\left(x_{i, n-m}\right)=q^{-1} x_{i, n-m}$. Consequently, $y_{2} x_{i j}=q x_{i j} y_{2}$ for $1 \leq i \leq m$ and $1 \leq j<n-m$, while $y_{2} x_{i, n-m}=q^{-1} x_{i, n-m} y_{2}$. 
Proof. When $j<n-m$, the calculations are similar to those in the proof of the previous result and so are omitted.

Set $N:=\{2, \ldots, m+1\} \backslash\{m+2-i\}$. Then, $x_{i, n-m}\left[M_{2}\right]=[N \cup\{1\}]$ and $\left[M_{2}\right]=[N \cup\{m+2-i\}]$. Now, $1<m+2-i$ so that $[1][m+2-i]=q[m+2-i][1]$ in $\mathcal{O}_{q}(G(1, n))$. By using Proposition 2.3, it follows that $\left(x_{i, n-m}\left[M_{2}\right]\right)\left[M_{2}\right]=$ $q\left[M_{2}\right]\left(x_{i, n-m}\left[M_{2}\right]\right)$. Hence, $x_{i, n-m}\left[M_{2}\right]=q\left[M_{2}\right] x_{i, n-m}$, and so $\sigma_{2}\left(x_{i, n-m}\right)=$ $q^{-1} x_{i, n-m}$ and $y_{2} x_{i, n-m}=q^{-1} x_{i, n-m} y_{2}$, as claimed.

\section{TWisting By A 2-COCYClE}

Given a $\mathbb{K}$-algebra $A$ that is graded by a semigroup, one can twist the multiplication in $A$ by using a 2 -cocycle to produce a new multiplication. We only need to deal with $\mathbb{Z}^{n}$-graded algebras, so we restrict our discussion to this case. For a general discussion of the process of twisting by 2-coycles, see, for example, 1 , Section 3].

Definition 5.1. A 2-cocycle (with values in $\mathbb{K}^{*}$ ) on $\mathbb{Z}^{n}$ is a map $c: \mathbb{Z}^{n} \times \mathbb{Z}^{n} \longrightarrow \mathbb{K}^{*}$ such that

$$
c(s, t+u) c(t, u)=c(s, t) c(s+t, u)
$$

for all $s, t, u \in \mathbb{Z}^{n}$.

Given a $\mathbb{Z}^{n}$-graded $\mathbb{K}$-algebra $A$, if $a$ is a homogeneous element in $A_{s}$, for $s \in \mathbb{Z}^{n}$, then we set content $(a):=s$.

Given a $\mathbb{Z}^{n}$-graded $\mathbb{K}$-algebra $A$ and a 2-cocycle $c$ on $\mathbb{Z}^{n}$, one can define a new $\mathbb{K}$-algebra $T(A)$ in the following way. As a graded vector space, $A$ and $T(A)$ are isomorphic via an isomorphism $a \mapsto a^{\prime}$. The multiplication in $T(A)$ is given by

$$
a^{\prime} b^{\prime}:=c(s, t)(a b)^{\prime}
$$

for homogeneous elements $a, b \in A$ with content $s$ and $t$, respectively. The defining condition of a 2-cocycle is precisely the condition needed to ensure that this multiplication is associative. We refer to $T(A)$ as the twist of $A$ by $c$, and the map $a \mapsto a^{\prime}$ is the twist map.

The property of being an integral domain is preserved under twists, as the next lemma shows.

Lemma 5.2. Let $A$ be a $\mathbb{Z}^{n}$-graded $\mathbb{K}$-algebra that is an integral domain, and let $c$ be a 2 -cocycle on $\mathbb{Z}^{n}$. Then $T(A)$ is an integral domain.

Proof. The algebra $T(A)$ is also $\mathbb{Z}^{n}$-graded, and we may consider $\mathbb{Z}^{n}$ as a totally ordered group. In order to see that the product of two nonzero elements $a^{\prime}, b^{\prime}$ of $T(A)$ is nonzero, it suffices to show that the product of their highest terms is nonzero. Hence, we may assume that $a, b$ are homogeneous elements. In this case, $a^{\prime} b^{\prime}$ is a nonzero scalar multiple of $(a b)^{\prime}$ and $a b \neq 0$, since $A$ is a domain. Hence, $T(A)$ is a domain, as required.

Our aim is to twist the quantum grassmannian $\mathcal{O}_{q}(G(m, n))$ by a suitable 2cocycle in such a way that the effect of the twist map is to cycle the indices of the generating quantum minors. There is a technical problem associated with this attempt, in that the defining relations for the quantum grassmannian (quantum Plücker relations) are complicated to deal with. We avoid the problem by using the notion of noncommutative dehomogenisation introduced earlier. 
Let the standard basis of $\mathbb{Z}^{n}$ be denoted by $\{\epsilon(1), \ldots, \epsilon(n)\}$ so that $\left(s_{1}, \ldots, s_{n}\right)$ denotes the element $s_{1} \epsilon(1)+\cdots+s_{n} \epsilon(n)$.

The quantum grassmannian $\mathcal{O}_{q}(G(m, n))$ has a natural grading by $\mathbb{Z}^{n}$ determined by the content of a generating quantum minor, where content $([I]):=\sum_{i \in I} \epsilon(i)$.

Note that $\left[M_{\alpha}\right]$ is a homogeneous element of $\mathcal{O}_{q}(G(m, n))$, and so the $\mathbb{Z}^{n}$-grading of $\mathcal{O}_{q}(G(m, n))$ extends in a natural way to $\mathcal{O}_{q}(G(m, n))\left[\left[M_{\alpha}\right]^{-1}\right]$ and hence to $\mathbb{K}\left[x_{i j}\right]\left[y_{\alpha}^{ \pm 1} ; \sigma_{\alpha}\right]$ by using the dehomogenisation isomorphism of Theorem 4.1]

Lemma 5.3. Let $p=q^{2 / m}$. The map $c: \mathbb{Z}^{n} \times \mathbb{Z}^{n} \longrightarrow \mathbb{K}^{*}$, defined by

$$
c\left(\left(s_{1}, \ldots, s_{n}\right),\left(t_{1}, \ldots, t_{n}\right)\right):=\prod_{j \neq n} p^{s_{n} t_{j}},
$$

is a 2-cocycle.

Proof. Set $s=\left(s_{1}, \ldots, s_{n}\right), t=\left(t_{1}, \ldots, t_{n}\right)$ and $u=\left(u_{1}, \ldots, u_{n}\right)$. We have to check that

$$
c(s, t+u) c(t, u)=c(s, t) c(s+t, u) .
$$

The proof is routine; one checks that each side is equal to

$$
\prod_{j \neq n} p^{s_{n} t_{j}+s_{n} u_{j}+t_{n} u_{j}} .
$$

Now, we look at the effect of twisting the algebra $A:=\mathbb{K}\left[x_{i j}\right]\left[y_{1}^{ \pm 1} ; \sigma_{1}\right]$ by using the 2-cocycle $c$. Write $y$ and $\sigma$ for $y_{1}$ and $\sigma_{1}$, respectively.

We denote by $T(A)$ the twist of $A$ by using the 2-cocyle $c$ so that if $a, b$ are homogeneous elements with content $s=\left(s_{1}, \ldots, s_{n}\right)$ and $t=\left(t_{1}, \ldots, t_{n}\right)$, respectively, then

$$
a^{\prime} b^{\prime}:=c\left(\left(s_{1}, \ldots, s_{n}\right),\left(t_{1}, \ldots, t_{n}\right)\right)(a b)^{\prime} .
$$

Now, we are in the case that $\alpha=1$ so that

$$
x_{i j}=[\{1, \ldots, m\} \cup\{j+m\} \backslash\{m+1-i\}][1, \ldots, m]^{-1} .
$$

Note that the content of $x_{i j}$ is $\epsilon(j+m)-\epsilon(m+1-i)$ and that the content of $y$ is $\epsilon(1)+\cdots+\epsilon(m)$.

As $A$ is generated by the homogeneous elements $x_{i j}$ and $y^{ \pm 1}$, the twisted algebra $T(A)$ is generated by the homogeneous elements $x_{i j}^{\prime}$ and $\left(y^{\prime}\right)^{ \pm 1}$. Our first aim is to describe the commutation relations satisfied by these elements.

We will often abuse notation by writing $c(a, b)$ instead of $c(\operatorname{content}(a), \operatorname{content}(b))$ for homogeneous elements $a, b \in A$.

Note that the value taken by $c$ on a pair of elements from the set $\left\{x_{i j}, y\right\}$ is often equal to $p^{0}=1$. In fact, the only possibilities for a value other than $p^{0}$ occur in the cases when $\epsilon(n)$ occurs in the content of the first argument in $c$. This can only occur for $x_{i, n-m}$, and we check that

$$
c\left(x_{i, n-m}, x_{l, n-m}\right)=p^{-1}, \quad c\left(x_{i, n-m}, y\right)=p^{m}=q^{2},
$$

while $c\left(x_{i, n-m}, x_{l, j}\right)=1$ for $j<n-m$ and $c\left(y, x_{i j}\right)=1$ for all $i, j$. These observations make the calculation of the twisted product on pairs from the set $\left\{x_{i j}^{\prime}, y^{\prime}\right\}$ very easy.

Lemma 5.4. $\left(x_{i j}^{\prime}\right)$ is a generic q-quantum matrix so that the algebra $\mathbb{K}\left[x_{i j}^{\prime}\right]$ is isomorphic to $\mathcal{O}_{q}\left(M_{m, n-m}\right)$. Moreover

$$
y^{\prime} x_{i j}^{\prime}=q x_{i j}^{\prime} y^{\prime} \text { for } j<n-m \quad \text { and } \quad y^{\prime} x_{i, n-m}^{\prime}=q^{-1} x_{i, n-m}^{\prime} y^{\prime} .
$$


Proof. First, we show that the $x_{i j}^{\prime}$ satisfy the commutation relations for a $q$ quantum matrix. The cases where $c(-,-)$ takes value 1 are easy to check. For example, for $i_{1}<i_{2}$ and $j<n-m$,

$$
x_{i_{1} j}^{\prime} x_{i_{2} j}^{\prime}=c\left(x_{i_{1} j}, x_{i_{2} j}\right)\left(x_{i_{1} j} x_{i_{2} j}\right)^{\prime}=\left(x_{i_{1} j} x_{i_{2} j}\right)^{\prime}
$$

while

$$
x_{i_{2} j}^{\prime} x_{i_{1} j}^{\prime}=c\left(x_{i_{2} j}, x_{i_{1} j}\right)\left(x_{i_{2} j} x_{i_{1} j}\right)^{\prime}=\left(x_{i_{2} j} x_{i_{1} j}\right)^{\prime}=q^{-1}\left(x_{i_{1} j} x_{i_{2} j}\right)^{\prime}=q^{-1} x_{i_{1} j}^{\prime} x_{i_{2} j}^{\prime},
$$

and so $x_{i_{1} j}^{\prime} x_{i_{2} j}^{\prime}=q x_{i_{2} j}^{\prime} x_{i_{1} j}^{\prime}$, as required.

Also, for $i_{1}<i_{2}$,

$$
x_{i_{1}(n-m)}^{\prime} x_{i_{2}(n-m)}^{\prime}=p^{-1}\left(x_{i_{1}(n-m)} x_{i_{2}(n-m)}\right)^{\prime}
$$

and

$$
x_{i_{2}(n-m)}^{\prime} x_{i_{1}(n-m)}^{\prime}=p^{-1}\left(x_{i_{2}(n-m)} x_{i_{1}(n-m)}\right)^{\prime},
$$

so again the desired $q$-commutation follows and the column relations are established.

The row relation computations are similar and so are omitted.

When $i_{1}<i_{2}$ and $j_{1}<j_{2}$, note that $c\left(x_{i_{1} j_{2}}, x_{i_{2} j_{1}}\right)=c\left(x_{i_{2} j_{1}}, x_{i_{1} j_{2}}\right)=1$, and so

$$
x_{i_{1} j_{2}}^{\prime} x_{i_{2} j_{1}}^{\prime}=\left(x_{i_{1} j_{2}} x_{i_{2} j_{1}}\right)^{\prime}=\left(x_{i_{2} j_{1}} x_{i_{1} j_{2}}\right)^{\prime}=x_{i_{2} j_{1}}^{\prime} x_{i_{1} j_{2}}^{\prime} \text {, }
$$

as required.

Continuing with $i_{1}<i_{2}$ and $j_{1}<j_{2}$, note that $c\left(x_{i_{1} j_{1}}, x_{i_{2} j_{2}}\right)=c\left(x_{i_{2} j_{2}}, x_{i_{1} j_{1}}\right)=1$, and so

$$
\begin{aligned}
x_{i_{1} j_{1}}^{\prime} x_{i_{2} j_{2}}^{\prime}-x_{i_{2} j_{2}}^{\prime} x_{i_{1} j_{1}}^{\prime} & =\left(x_{i_{1} j_{1}} x_{i_{2} j_{2}}\right)^{\prime}-\left(x_{i_{2} j_{2}} x_{i_{1} j_{1}}\right)^{\prime}=\left(x_{i_{1} j_{1}} x_{i_{2} j_{2}}-x_{i_{2} j_{2}} x_{i_{1} j_{1}}\right)^{\prime} \\
& =\left(q-q^{-1}\right)\left(x_{i_{1} j_{2}} x_{i_{2} j_{1}}\right)^{\prime}=\left(q-q^{-1}\right) x_{i_{1} j_{2}}^{\prime} x_{i_{2} j_{1}}^{\prime} .
\end{aligned}
$$

This finishes the verification that the $x_{i j}^{\prime}$ satisfy the commutation relations of $\mathcal{O}_{q}\left(M_{m, n-m}\right)$. As a result, there is a homorphism $\phi$ from $\mathcal{O}_{q}\left(M_{m, n-m}\right)=\mathbb{K}\left[x_{i j}\right]$ onto $\mathbb{K}\left[x_{i j}^{\prime}\right]$ that sends $x_{i j}$ to $x_{i j}^{\prime}$. As the lexicographically ordered monomials $x_{i_{1} j_{1}} x_{i_{2} j_{2}} \ldots x_{i_{t} j_{t}}$ are linearly independent, so are their scalar multiples $x_{i_{1} j_{1}}^{\prime} x_{i_{2} j_{2}}^{\prime} \ldots$ $x_{i_{t} j_{t}}^{\prime}$. It follows that the homomorphism $\phi$ is an isomorphism.

Now, we calculate how $y^{\prime}$ commutes with the $x_{i j}^{\prime}$.

For $j<n-m$, observe that

$$
x_{i j}^{\prime} y^{\prime}=c\left(x_{i j}, y\right)\left(x_{i j} y\right)^{\prime}=\left(x_{i j} y\right)^{\prime},
$$

and so

$$
\begin{aligned}
y^{\prime} x_{i j}^{\prime} & =c\left(y, x_{i j}\right)\left(y x_{i j}\right)^{\prime}=\left(y x_{i j}\right)^{\prime}=q\left(x_{i j} y\right)^{\prime} \\
& =q x_{i j}^{\prime} y^{\prime} .
\end{aligned}
$$

Finally,

$$
x_{i, n-m}^{\prime} y^{\prime}=c\left(x_{i, n-m}, y\right)\left(x_{i, n-m} y\right)^{\prime}=q^{2}\left(x_{i, n-m} y\right)^{\prime},
$$

and so

$$
y^{\prime} x_{i, n-m}^{\prime}=c\left(y, x_{i, n-m}\right)\left(y x_{i, n-m}\right)^{\prime}=\left(y x_{i, n-m}\right)^{\prime}=q\left(x_{i, n-m} y\right)^{\prime}=q^{-1} x_{i, n-m}^{\prime} y^{\prime} .
$$

We now wish to consider the dehomogenisation isomorphism when $\alpha=2$. In order to avoid a clash of notation, we will write

$$
\mathcal{O}_{q}(G(m, n))\left[\left[M_{2}\right]^{-1}\right] \cong \mathbb{K}\left[z_{i j}\right]\left[w^{ \pm 1} ; \phi\right],
$$

where $z_{i j}:=\left[M_{2} \cup\{j \widetilde{+m+1}\} \backslash\{m+2-i\}\right]$ and $M_{2}=[2,3, \ldots, m+1]$. 


\section{Theorem 5.5.}

$$
T\left(\mathbb{K}\left[x_{i j}\right]\left[y^{ \pm 1} ; \sigma\right]\right) \cong \mathbb{K}\left[z_{i j}\right]\left[w^{ \pm 1} ; \phi\right]
$$

via a map $\theta: T\left(\mathbb{K}\left[x_{i j}\right]\left[y^{ \pm 1} ; \sigma\right]\right) \longrightarrow \mathbb{K}\left[z_{i j}\right]\left[w^{ \pm 1} ; \phi\right]$, which sends $x_{i j}^{\prime}$ to $z_{i j}$ and $y$ to $w$.

Proof. From Lemma 4.4 and Lemma 5.4, we see that the commutation relations between the elements of the generating set $\left\{x_{i j}^{\prime}, y^{\prime}\right\}$ of $T\left(\mathbb{K}\left[x_{i j}\right]\left[y^{ \pm 1} ; \sigma\right]\right)$ are the same as the corresponding commutation relations among the elements of the generating set $\left\{z_{i j}^{\prime}, y_{2}^{\prime}\right\}$ of $\mathbb{K}\left[z_{i j}\right]\left[y_{2}^{ \pm 1} ; \sigma_{2}\right]$.

Thus, we may define a homomorphism from $\mathbb{K}\left[z_{i j}\right]\left[w^{ \pm 1} ; \phi\right]$ to $T\left(\mathbb{K}\left[x_{i j}\right]\left[y^{ \pm 1} ; \sigma\right]\right)$ by sending $z_{i j}$ to $x_{i j}^{\prime}$ and $w$ to $y^{\prime}$. As the lexicographically ordered monomials $x_{i_{1} j_{1}} x_{i_{2} j_{2}} \ldots x_{i_{t} j_{t}} y^{k}$ are linearly independent, so are their scalar multiples $x_{i_{1} j_{1}}^{\prime} x_{i_{2} j_{2}}^{\prime}$ $\ldots x_{i_{t} j_{t}}^{\prime} y^{\prime k}$. It follows that this homomorphism is an isomorphism.

We may identify $\mathcal{O}_{q}(G(m, n))$ as a subalgebra of $\mathbb{K}\left[x_{i j}\right]\left[y^{ \pm 1} ; \sigma\right]$ via the dehomogenisation isomorphism $\mathcal{O}_{q}(G(m, n))\left[\left[M_{1}\right]^{-1}\right] \cong \mathbb{K}\left[x_{i j}\right]\left[y^{ \pm 1} ; \sigma\right]$ and identify another copy of $\mathcal{O}_{q}(G(m, n))$ with a subalgebra of $\mathbb{K}\left[z_{i j}\right]\left[y_{2}^{ \pm 1} ; \sigma_{2}\right]$ via the isomorphism $\mathcal{O}_{q}(G(m, n))\left[\left[M_{2}\right]^{-1}\right] \cong \mathbb{K}\left[z_{i j}\right]\left[y_{2}^{ \pm 1} ; \sigma_{2}\right]$. Our next aim is to show that the image of the first copy of $\mathcal{O}_{q}(G(m, n))$ under the map $\theta \circ T$ is the second copy of $\mathcal{O}_{q}(G(m, n))$. In order to do this, we need to track the image of a generating quantum minor through the sequence of maps

$$
\begin{aligned}
\mathcal{O}_{q}(G(m, n)) \stackrel{\phi_{1}}{\longrightarrow} \mathbb{K}\left[x_{i j}\right]\left[y_{1}^{ \pm 1} ; \sigma_{1}\right] & \stackrel{T}{\longrightarrow} \mathbb{K}\left[x_{i j}^{\prime}\right]\left[y_{1}^{\prime \pm 1}\right] \\
& \stackrel{\theta}{\longrightarrow} \mathbb{K}\left[z_{i j}\right]\left[y_{2}^{ \pm 1} ; \sigma_{2}\right] \stackrel{\rho_{2}}{\longrightarrow} \mathcal{O}_{q}(G(m, n))\left[\left[M_{2}\right]^{-1}\right] .
\end{aligned}
$$

First, we record the effect of the twist map on quantum minors. We need to consider quantum minors in each of the quantum matrix algebras $\mathbb{K}\left[x_{i j}\right]$ and $\mathbb{K}\left[x_{i j}^{\prime}\right]$, so for a given row set $I$ and column set $J$ we will denote the corresponding quantum minors by $[I \mid J]_{x}$ and $[I \mid J]_{x^{\prime}}$, respectively.

Lemma 5.6. Let $[I \mid J]_{x}$ be a quantum minor of the quantum matrix algebra $\mathbb{K}\left[x_{i j}\right]$ in the previous theorem. Then the image of $[I \mid J]_{x}$ under the twist map is $[I \mid J]_{x^{\prime}}$.

Proof. This proof is a routine calculation, using the fact that each $c(-,-)$ that occurs takes value 1 to observe that $\left(x_{i_{\sigma(1)} j_{1}} \cdots x_{i_{\sigma(t)} j_{t}}\right)^{\prime}=x_{i_{\sigma(1)} j_{1}} \cdots x_{i_{\sigma(t)} j_{t}}$.

Lemma 5.7. $c([I \mid J], y)=1$ when $n-m \notin J$ and $c([I \mid J], y)=q^{2}$ when $n-m \in J$.

Proof. This follows from the fact that $\epsilon(n)$ appears (with nonzero coefficients) in content $([I \mid J])$ if and only if $n-m \in J$ by [14, Proposition 4.3].

As before, for a given row set $I$ and column set $J$, we will denote the corresponding quantum minors of the various quantum matrix algebras by $[I \mid J]_{x},[I \mid J]_{x^{\prime}}$ and $[I \mid J]_{z}$, respectively.

Lemma 5.8. Let $I=\left[i_{1}, \ldots, i_{m}\right]$ be a generating quantum minor of $\mathcal{O}_{q}(G(m, n))$. Then

$$
\rho_{2} \circ \theta \circ T \circ \phi_{1}([I])=\left\{\begin{array}{cl}
{\left[i_{1}+1, \ldots, i_{m}+1\right]} & \text { if } i_{m} \neq n, \\
q^{-2}\left[1, i_{1}+1, \ldots, i_{m-1}+1\right] & \text { if } i_{m}=n .
\end{array}\right.
$$


Proof. Note that

$$
\phi_{1}([I])=\left[(m+1)-\left(M_{1} \backslash I_{r}\right) \mid I_{c}-m\right]_{x} y_{1},
$$

and so

$T \circ \phi_{1}([I])=\left(\left[(m+1)-\left(M_{1} \backslash I_{r}\right) \mid I_{c}-m\right]_{x} y_{1}\right)^{\prime}=C^{-1}\left[(m+1)-\left(M_{1} \backslash I_{r}\right) \mid I_{c}-m\right]_{x^{\prime}} y_{1}^{\prime}$, where $C:=c\left(\left[(m+1)-\left(M_{1} \backslash I_{r}\right) \mid I_{c}-m\right]_{x}, y\right)$. Also note that $C=1$ if $n-m \notin I_{c}-m$ (and so if $n \notin I$ ), while $C=q^{2}$ if $n-m \in I_{c}-m$ (and so if $n \in I$ ). Thus,

$$
\begin{aligned}
\theta \circ T \circ \phi_{1}(I) & =C^{-1}\left[(m+1)-\left(M_{1} \backslash I_{r}\right) \mid I_{c}-m\right]_{z} w \\
& =C^{-1}\left[(m+2)-\left(M_{2} \backslash\left(I_{r}+1\right)\right) \mid\left(I_{c}+1\right)-(m+1)\right]_{z} w .
\end{aligned}
$$

Finally,

$$
\begin{aligned}
\rho_{2} \circ \theta \circ T \circ \phi_{1}(I) & =C^{-1} \rho_{2}\left(\left[(m+2)-M_{2} \backslash\left(I_{r}+1\right) \mid\left(I_{c}+1\right)-(m+1)\right]_{z} w\right) \\
& =C^{-1}\left[\left(I_{r}+1\right) \sqcup\left(I_{c}+1\right)\right]=C^{-1}[I+1],
\end{aligned}
$$

and the result follows. Note that the penultimate equality is obtained by the same calculation as in the proof of Corollary 4.2 .

We can now reach our conclusion.

Theorem 5.9.

$$
T\left(\mathcal{O}_{q}(G(m, n))\right) \cong \mathcal{O}_{q}(G(m, n))
$$

via a map $\theta$ that sends $\left[i_{1}, \ldots, i_{m}\right]^{\prime}$ to $\left[i_{1}+1, \ldots, i_{m}+1\right]$, for $i_{m}<n$, and sends $\left[i_{1}, \ldots, i_{m-1}, n\right]^{\prime}$ to $q^{-2}\left[1, i_{1}+1, \ldots, i_{m-1}+1\right]$.

Proof. This follows immediately from the previous lemma.

\section{Twisting the $\mathcal{H}$-PRIME SPECTRUm}

In this section we assume that $q$ is a not a root of unity in order that we know that the prime ideals of $\mathcal{O}_{q}(G(m, n))$ are completely prime; see [11, Theorem 5.2].

The natural $\mathbb{Z}^{n}$-grading on $\mathcal{O}_{q}(G(m, n))$ induces a rational action of the algebraic torus $\mathcal{H}:=\left(\mathbb{K}^{*}\right)^{n}$ on $\mathcal{O}_{q}(G(m, n))$ by $\mathbb{K}$-automorphisms via

$$
\left(h_{1}, \ldots, h_{n}\right) \cdot\left[i_{1}, \ldots, i_{m}\right]=h_{i_{1}} \cdots h_{i_{m}}\left[i_{1}, \ldots, i_{m}\right]
$$

(see [2, Lemma II.2.11] for more details). In this setting, the homogeneous prime ideals of $\mathcal{O}_{q}(G(m, n))$ are exactly those primes that are invariant under this torus action. Hence homogeneous primes are also called $\mathcal{H}$-primes, and the set $\mathcal{H}-$ $\operatorname{Spec}\left(\mathcal{O}_{q}(G(m, n))\right)$ of all $\mathcal{H}$-primes of $\mathcal{O}_{q}(G(m, n))$ is called the $\mathcal{H}$-prime spectrum of $\mathcal{O}_{q}(G(m, n))$. It was proved in [11] that this set is finite, and its cardinality was computed. The importance of the $\mathcal{H}$-prime spectrum was pointed out by Goodearl and Letzter, who proved that the $\mathcal{H}$-prime spectrum parametrizes a natural stratification of the prime spectrum of $\mathcal{O}_{q}(G(m, n))$. We continue to use the twist map $T$ determined by the 2-cocycle from the previous section. Note that this twist map is $\mathcal{H}$-equivariant.

Theorem 6.1. Suppose that $q$ is not a root of unity. Let $P$ be an $\mathcal{H}$-prime ideal of $\mathcal{O}_{q}(G(m, n))$. Then $T(P):=\left\{p^{\prime} \mid p \in P\right\}$ is an $\mathcal{H}$-prime ideal of $T\left(\mathcal{O}_{q}(G(m, n))\right)$.

Proof. The algebra $\mathcal{O}_{q}(G(m, n)) / P$ inherits a $\mathbb{Z}^{n}$-grading, as $P$ is homogeneous, and so we can form the twisted algebra $T\left(\mathcal{O}_{q}(G(m, n)) / P\right)$. It then follows that $T\left(\mathcal{O}_{q}(G(m, n)) / P\right) \cong T\left(\mathcal{O}_{q}(G(m, n))\right) / T(P)$. Hence, it is enough to show that $T\left(\mathcal{O}_{q}(G(m, n)) / P\right)$ is a domain, and this follows from Lemma 5.2 . 
The map $\theta$ defined in Theorem 5.9 above is not $\mathcal{H}$-equivariant. However, it is easy to check that if $h=\left(h_{1}, \ldots, h_{n-1}, h_{n}\right) \in \mathcal{H}$ and we set $h^{\prime}=\left(h_{n}, h_{1}, \ldots, h_{n-1}\right) \in \mathcal{H}$, then $h^{\prime} \circ \theta=\theta \circ h$. From this it follows that $\mathcal{H}$-prime ideals are sent to $\mathcal{H}$-prime ideals by $\theta$.

Corollary 6.2. Suppose that $q$ is not a root of unity. Then

$$
\theta\left(T\left(\mathcal{H}-\operatorname{Spec}\left(\mathcal{O}_{q}(G(m, n))\right)\right)\right)=\mathcal{H}-\operatorname{Spec}\left(\mathcal{O}_{q}(G(m, n))\right),
$$

where $\theta$ is the isomorphism defined in Theorem 5.9 .

Proof. If $P, Q$ are two distinct $\mathcal{H}$-prime ideals of $\mathcal{O}_{q}(G(m, n))$, then $T(P)$ and $T(Q)$ are distinct $\mathcal{H}$-prime ideals of $T\left(\mathcal{O}_{q}(G(m, n))\right)$; and so their images under the isomorphism $\theta$ are distinct $\mathcal{H}$-prime ideals of $\mathcal{O}_{q}(G(m, n))$. As the set of $\mathcal{H}$-prime ideals is finite, this establishes the claim.

It follows that if $P$ is an $\mathcal{H}$-prime ideal of $\mathcal{O}_{q}(G(m, n))$, then a quantum minor $\left[i_{1}, \ldots, i_{m}\right]$ is in $P$ if and only if the quantum minor $\left[i_{1}+1, \ldots, i_{m}+1\right]$ is in $\theta(T(P))$, where $i_{m}+1:=1$ if $i_{m}=n$.

Note that in [11] it was shown that each $\mathcal{H}$-prime ideal of $\mathcal{O}_{q}(G(2,4))$ is generated by the quantum minors that it contains, and it was conjectured that this holds in any $\mathcal{O}_{q}(G(m, n))$.

\section{ACKNOWLEDGEMENT}

We thank the anonymous referee for comments that have improved the presentation of this paper.

\section{REFERENCES}

[1] M Artin, W Schelter and J Tate, Quantum deformations of $\mathrm{GL}_{n}$, Comm. Pure Appl. Math. 44 (1991), no. 8-9, 879-895. MR1127037 (92i:17014)

[2] K A Brown and K R Goodearl, Lectures on Algebraic Quantum Groups, Advanced Courses in Mathematics. CRM Barcelona, Birkhäuser Verlag, Basel, 2002. MR.1898492 (2003f:16067)

[3] K R Goodearl, S Launois and T H Lenagan, Totally nonnegative cells and matrix Poisson varieties, arXiv:0905.3631.

[4] K R Goodearl, S Launois and T H Lenagan, Torus-invariant prime ideals in quantum matrices, totally nonnegative cells and symplectic leaves, arXiv:0909.3935. Mathematische Zeitschrift, doi:10.1007/s00209-010-0714-5.

[5] K R Goodearl and T H Lenagan, Quantum determinantal ideals, Duke Math. J. 103 (2000), 165-190. MR.1758243 (2001k:16080)

[6] K R Goodearl and M Yakimov, Poisson structures on affine spaces and flag varieties. II, Trans. Amer. Math. Soc. 361 (2009), 5753-5780. MR 2529913

[7] A Kelly, T H Lenagan, and L Rigal, Ring theoretic properties of quantum grassmannians, J. Algebra Appl. 3 (2004), no. 1, 9-30. MR2047633 (2005b:20096)

[8] G R Krause and T H Lenagan, Growth of algebras and Gelfand-Kirillov dimension, Graduate Studies in Mathematics, 22. American Mathematical Society, Providence, RI, 2000. MR $1721834(2000 \mathrm{j}: 16035)$

[9] D Krob and B Leclerc, Minor identities for quasi-determinants and quantum determinants, Comm. Math. Phys. 169 (1995), no. 1, 1-23. MR1328259 (96g:15015)

[10] A Knutson, T Lam and D E Speyer, Positroid varieties I: juggling and geometry, arXiv:0903.3694.

[11] S Launois, T H Lenagan and L Rigal, Prime ideals in the quantum grassmannian, Selecta Mathematica 13 (2008), 697-725. MR2403308 (2009e:20110)

[12] T H Lenagan and L Rigal, Quantum graded algebras with a straightening law and the ASCohen-Macaulay property for quantum determinantal rings and quantum grassmannians, J. Algebra 301 (2006), no. 2, 670-702. MR2236763(2007g:16059) 
[13] T H Lenagan and L Rigal, Quantum analogues of Schubert varieties in the grassmannian, Glasgow Math. J. 50 (2008), no. 1, 55-70. MR2381732 (2008m:20080)

[14] T H Lenagan and E J Russell, Cyclic orders on the quantum grassmannian, Arabian Journal for Science and Engineering 33 (2008), 337-350. MR2500045

[15] A Postnikov, Total positivity, Grassmannians, and networks, arXiv:0609764.

[16] M Yakimov, Cyclicity of Lusztig's stratification of grassmannians and Poisson geometry, In: Noncommutative Structures in Mathematics and Physics, eds. S. Caenepeel, J. Fuchs, S. Gutt, Ch. Schweigert, A. Stolin, and F. van Oystaeyen, pp. 258-262, Royal Flemish Academy of Belgium for Sciences and Arts, 2010.

School of Mathematics, Statistics and Actuarial Science, University of Kent, CanTerbury, Kent CT2 7NF, United Kingdom

E-mail address: S.Launois@kent.ac.uk

Maxwell Institute for Mathematical Sciences, School of Mathematics, University of Edinburgh, James Clerk Maxwell Building, King's Buildings, Mayfield Road, EdinBURGH EH9 3JZ, UNITED KINGDOM

E-mail address: tom@maths.ed.ac.uk 